\title{
Flow patterns and preferred sites of atherosclerotic lesions in the human aorta - I. Aortic arch
}

\author{
Shunsuke Endo ${ }^{\mathrm{a}, *}$, Harry L. Goldsmith ${ }^{\mathrm{b}}$ and Takeshi Karino ${ }^{\mathrm{c}}$ \\ ${ }^{a}$ Department of General Thoracic Surgery, Jichi Medical University, Tochigi, Japan \\ ${ }^{\mathrm{b}}$ Department of Medicine, McGill University Health Centre, Montreal, QC, Canada \\ ${ }^{\mathrm{c}}$ Research Institute for Electronic Science, Hokkaido University, Sapporo, Japan
}

Received 5 February 2014

Accepted in revised form 31 July 2014

\begin{abstract}
.
OBJECTIVES: To elucidate the role of fluid mechanical factors in the localized genesis and development of atherosclerotic lesions in man.

METHODS: Flow patterns and preferred sites of atherosclerotic lesions in the human aortic arch were studied in detail using isolated transparent aortic trees prepared from humans postmortem and by means of flow visualization of tracer polystyrene microspheres, using cinemicrographic techniques.

RESULTS: Under the condition of steady flow that simulated mid-systole, the flow in the aortic arch consisted of three major components; (i) a straight flow to the brachio-cephalic artery located close to the right dorsal wall of the ascending aorta; (ii) a quasi-parallel undisturbed flow located close to the common median plane of the aortic arch and its side branches, and (iii) a clockwise slow, spiral secondary flow located dominantly near the left ventral wall of the aortic arch. Thus, looking down the aorta from its origin, the flow in the aortic arch appeared as a single helical flow revolving in a clockwise direction.

CONCLUSIONS: Atherosclerotic lesions were found mainly in regions of low wall shear stress such as the proximal lip of the orifice of each side branch where a slow recirculation flow formed, and the left ventral wall of the aortic arch where a slow spiral secondary flow formed.
\end{abstract}

Keywords: Flow visualization, helical flow, recirculation flow, secondary flow, velocity distribution, wall shear stress

\section{Introduction}

Atherosclerosis is a degenerative disease that occurs in relatively large arteries by progressive thickening of the intima of the vessel wall, and its eventual hardening. Accumulation of low-density lipoproteins (LDL, a main carrier of cholesterol in flowing blood) within the intima, and migration of macrophages into subendothelial spaces are regarded as the two important steps that lead to the initiation of atherosclerosis [1-5]. It has been shown that atherosclerotic changes of an arterial wall in humans occur not randomly, and not everywhere in the arterial tree, but preferentially at certain sites such as the inner wall

\footnotetext{
*Address for correspondence: Dr. Shunsuke Endo, Department of General Thoracic Surgery, Jichi Medical University, 1-3311 Yakushiji, Shimotsuke, Tochigi 329-0498, Japan. Tel.: +81 28558 7368; Fax: +81 28544 6271; E-mail: tcvshun@jichi.ac.jp.
} 
of curved segments and outer walls of bifurcations of relatively large arteries. At such sites, due to sudden changes in direction of the flow and/or vessel diameter, blood flow is likely to be disturbed by the formation of secondary and recirculation flows, and endothelial cells are exposed to relatively low shear stresses [6-11]. One of the representative sites is the region of the aortic arch and the abdominal aorta that contains an acute bend and large diameter side branches. Therefore, extensive studies have been carried out in the past years on both the preferred sites of atherosclerotic lesions and the blood flow in this region. Localization of atherosclerotic lesions was studied by observing and analyzing the spatial distribution of fatty streaks and complicated lesions formed on the intimal surfaces of the aortas obtained from humans postmortem [6-11]. Blood flow was studied using various glass or plastic models, a cast of the human aorta [12-20], and natural vessels in living humans [21-29]. Measurements of fluid velocity were carried out mainly using ultrasound Doppler velocimeters $[18,19,22]$. Flow patterns were observed by injecting dyes [15,30], or tracking the particles added to the fluid as a tracer [6,14]. Furthermore, by the development of sophisticated diagnostic techniques such as echocardiography and magnetic resonance imaging (MRI), it has become possible to assess non-invasively the hemodynamics in various models of the human aorta as well as in real vessels in living humans, and display flow patterns, velocity profiles, and isovelocity contours [16,17,21,23,29,31]. It has also become possible to simulate the blood flow in the aortic tree by means of Computational Fluid Dynamics [12,31-38]. However, although there have been many studies on flow in the aortic arch, most of them were confined to the structure of the flow within the arch. No information has been provided on detailed flow patterns in the junction region of the three branches that we considered important in studying the relationship between blood flow and preferred sites of atherosclerotic lesions. One of the reasons for that appears to be due to the difficulty in making realistic models. Especially in the case of the aortic arch, it is not just a plane and curved vessel but a twisted and tapered bend with several major side branches coming off the outer wall in a region of high curvature. Another reason is that the flow visualization techniques mentioned above provided only fragmental information on the flow such as velocity profiles at certain cross-sections and dye streaks on only certain streamlines. To better understand the relationship between the blood flow and the sites of atherosclerotic lesions in the human aortic arch and the abdominal aorta, it was deemed necessary to study detailed 3-dimensional flow patterns and precise sites of atherosclerotic lesions in real vessels. Hence we have prepared isolated transparent aortic trees from humans postmortem by a method developed in our laboratory, and studied the relationship between the detailed flow patterns and the preferred sites of atherosclerotic lesions obtained with them. In this paper, the results obtained on the human aortic arch are described in detail. The results on the abdominal aorta will be presented in the succeeding paper.

\section{Materials and methods}

\subsection{Materials}

Thirty-eight human aortas were obtained at autopsy in the Department of Pathology, Montreal General Hospital, from 25 males and 13 females their ages ranging from 21 to 86 years (mean: 63 years old) fully one day after the death of the subject.

\subsection{Preparation of transparent aortic trees}

Out of the 38 aortas, 12 aortas including the heart and nine major branches were pressure-fixed and rendered transparent by modification of the method described by Karino and Motomiya [39]. 
After rinsing the vessel with isotonic saline and clearing surrounding tissues, a small opening was made to the left ventricle at its apex and a polyethylene tube having $9.8 \mathrm{~mm}$ i.d. and $12.3 \mathrm{~mm}$ o.d. was inserted into the bottom portion of the left ventricle aligning its axis as close as possible to that of the ascending aorta. The tube was firmly fixed to the heart by ligating the surrounding heart muscles onto it, and this provided an inlet for a fluid from a head tank to the aortic tree via the left ventricle. The pulmonary artery and veins were ligated, and then the nine major branches of the aortic tree (brachio-cephalic artery, left common carotid artery, left subclavian artery, celiac artery, superior mesenteric artery, left and right renal arteries, left and right common iliac arteries) were cannulated with thin-walled stainless-steel pipes or polyethylene tubes. All other branches that included the intercostal arteries, inferior mesenteric artery and lumbar arteries were occluded at their branching points by ligating with 6-0 Prolene suturing thread and coagulating them with a fulgurator.

The aortic tree was pressurized to the physiologic mean arterial blood pressure of $100 \mathrm{mmHg}$ by perfusing and immersing it in isotonic saline to stretch and set the vessel as close as possible to its natural state in vivo and then mounted on a three-dimensional stainless steel supporting frame by ligating the inflow tube and the cannula of each branch onto the arms of the supporting frame. The aortic tree with the heart was then histologically fixed for a few days by perfusing with and immersing it in a mixture of $2 \%$ glutaraldehyde and $4 \%$ formaldehyde in isotonic saline at a perfusion pressure of $100 \mathrm{mmHg}$.

The aortic tree was dehydrated for about one week by perfusing with, and immersing it in ethanolsaline mixtures of progressively increasing ethanol concentration under the same perfusion pressure, and finally suspended in pure ethanol. Here, the aortic valve was fixed at its fully opened position by placing a balloon catheter during the fixation and dehydration periods to simulate the condition in mid-systole. Finally, the aortic tree was filled with and immersed in oil of wintergreen (methyl salicylate) containing ethanol at $5 \%$ by volume under a perfusion pressure of $100 \mathrm{mmHg}$ to render the aortic tree transparent. Figure 1 shows the photographs of one of the aortic trees taken before (panel (a)) and after (panel (b)) it was rendered transparent.

The vessel prepared by this method lost the elasticity of the natural living arteries during the process of fixing, dehydrating, and rendering them transparent. However, the method did ensure the preservation of the complex three-dimensional configuration of the natural aortic tree that we considered the most important factor for studying the flow patterns.

Out of the 12 aortic trees pressure-fixed and rendered transparent, only 4 were actually used for flow studies. The other eight were not usable for flow studies due to severe atherosclerotic lesions since the thickened vessel wall and the calcified complicated lesions, that appeared as dark and black spots (cf. Fig. 2), prevented light transmission and made it difficult to trace the movements of tracer particles. They were used to collect information on spatial distributions of atherosclerotic lesions developed in these vessels. Twenty-six aortas that were not pressure-fixed were detached from the heart, cut and opened at the posterior wall and used to macroscopically locate the preferred sites of atherosclerotic lesions.

\subsection{Experimental procedures and analysis}

The transparent aortic tree was placed horizontally in a large glass chamber $(70 \mathrm{~cm} \times 20 \mathrm{~cm} \times 25 \mathrm{~cm})$ filled with the same liquid as that used to render the vessel transparent and trans-illuminated with a tungsten-filament lamp. The aortic tree was observed from various angles by changing its orientation relative to the direction of the light beams and photographed together with a ruler on $35 \mathrm{~mm}$ color or black and white films with a Nikon FE $35 \mathrm{~mm}$ camera. 


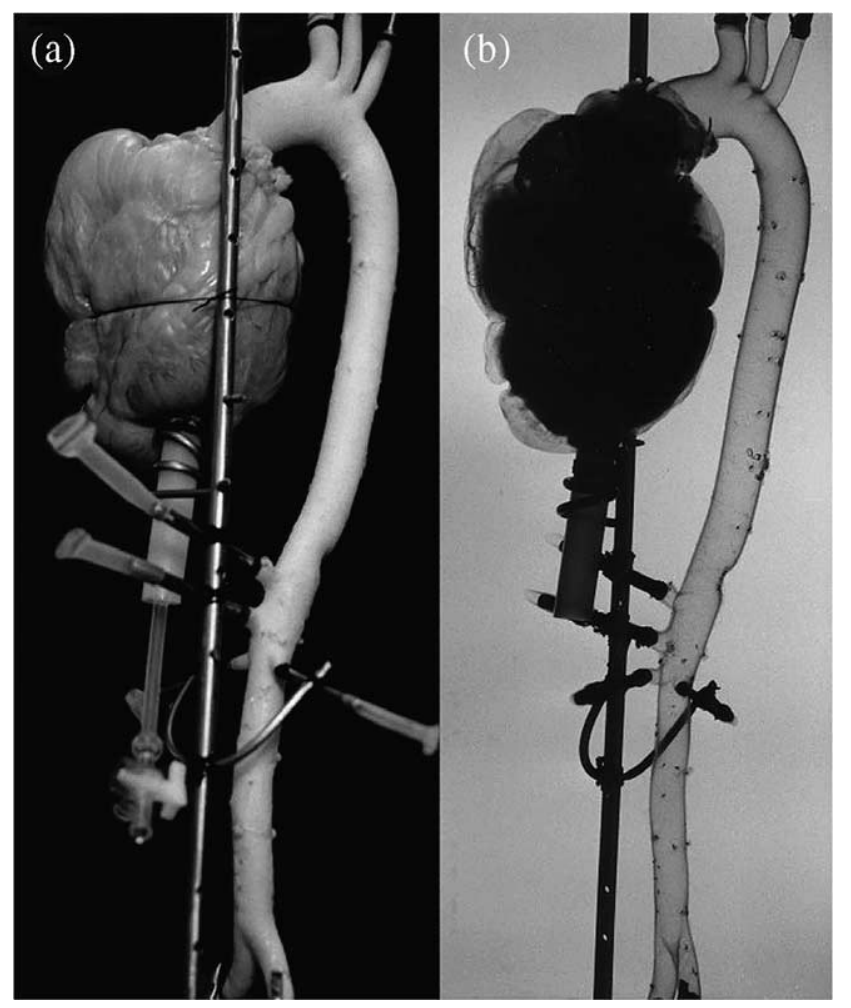

Fig. 1. (a) Photographs (left anterior oblique view) of a human aortic tree with the heart taken just after it was pressure-fixed and (b) after it was rendered transparent by perfusing with and immersing it in methyl salicylate containing 5\% ethanol, showing the geometric structure of the human aorta. A polyethylene tube inserted into the apex of the heart provides an inlet for fluid from head tank to aortic tree via the left ventricle.

The aortic tree in the glass chamber was then placed on a vertically movable horizontal stage located in front of the vertically mounted stage of an illumination system. The inlet tube inserted into the bottom portion of the left ventricle was connected to a 4 liter-capacity head tank via a $6.4 \mathrm{~mm}$ i.d. polyethylene tube. The outlet from each major branch was connected at its cannula to a collecting reservoir via a flexible polyethylene tube whose inner diameter was about the same as that of the cannula.

The areas of interest in the aortic tree were trans-illuminated with condensed parallel light from a tungsten filament lamp that was used mainly for observation, or high-intensity light from a $200 \mathrm{~W}$ d.c. mercury arc lamp with a blue filter to eliminate ultraviolet illumination that was used for filming.

We used oil of winter green (methyl salicylate) containing 5\% ethanol by volume to render the vessel transparent. Therefore, to keep the arterial tree transparent, we used the same liquid as a substitute for blood in our flow study. This liquid did not show non-Newtonian behavior like blood. However, in the present study, the Reynolds numbers for the flow of the liquid in the human aorta were kept within the range of physiological values of 6,100 (in young volunteers), and 4,200 (in age matched controls) at peak systole (estimated based on the data of Bürk et al. [21] obtained in 15 subjects). Furthermore, time-averaged wall shear rates assessed in any segment of the aorta were well over the critical value of $\sim 100 \mathrm{~s}^{-1}$ above which blood behaves as a Newtonian fluid. Therefore, we considered that any nonNewtonian characteristics of blood would have little effect on blood flow in the human aorta and hence blood could be substituted by a Newtonian fluid. 
Using the above liquid, a very dilute suspension of a mixture of 50, 80, 115, 230 and $330 \mu$ m-diameter polystyrene microspheres (density $\rho_{\mathrm{s}}=1.06 \mathrm{~g} / \mathrm{cm}^{3}$; Duke Scientific Corp., Palo Alto, CA) in oil of wintergreen with $5 \%$ ethanol (density $\rho=1.16 \mathrm{~g} / \mathrm{cm}^{3}$, viscosity $\mu=2.6 \mathrm{mPa}$ s, refractive index $\left.n_{\mathrm{D}}=1.53\right)$ was prepared and used as a substitute for blood. The reason why we used many different size particles as a tracer was, that due to the occurrence of radial migration of particles away from the vessel wall, only a few large size particles were found near the vessel wall and in the recirculation zones. Therefore, to avoid the defect, we made a suspension of various size particles.

The head tank and the whole aortic tree including the outflow tubes connected to each branch were filled with the suspension. Then the suspension was subjected to steady flow through the transparent aorta, and by adjusting the height of the head tank as well as each collecting reservoir, the volume flow rate ratio $Q_{\text {branch }} / Q_{\text {total }}$ was established within the physiological range under the resting conditions as follows: brachio-cephalic artery: 0.14; left common carotid artery: 0.08; left subclavian artery: 0.08; celiac and superior mesenteric artery: 0.15; right and left renal artery: each 0.12 , and right and left common iliac artery: each 0.08 .

The behavior of individual suspended tracer particles flowing through the aortic arch was observed through a zoom lens system attached to a cine-camera, and photographed on $16 \mathrm{~mm}$ cine-films at film speeds from 500 to 1,000 frames per second. To better understand the three dimensional flow patterns, the flow was observed and recorded from two different directions, that is, normal and parallel to the common median plane of the aortic arch and its three side branches by rotating the aortic tree.

The developed $35 \mathrm{~mm}$ films were projected with a slide projector onto a glass screen and analyzed to obtain inner diameters of the vessel. The developed $16 \mathrm{~mm}$ cine-films were subsequently projected onto a drafting table, and the movements of individual tracer particles were analyzed frame by frame with the aid of a stop-motion $16 \mathrm{~mm}$ movie analyzer (Vangurd Instrument Corp., Melville, NY) to obtain detailed flow patterns and distributions of fluid velocity and wall shear rate (shear stress).

The representative geometric and flow conditions such as the vessel diameter, $D_{\mathrm{o}}$, mean volume flow rate, $Q_{\mathrm{o}}$, mean fluid velocity, $\bar{U}$, and Reynolds number, $\operatorname{Re}_{\mathrm{o}}=D_{\mathrm{o}} \bar{U} \rho / \mu$, were evaluated in the middle portion of the ascending aorta. Here the cross-sectional shape of the aorta was not circular but elliptical. Therefore, the diameter of the vessel was calculated assuming the vessel cross-section to be an ellipse, taking the mean values of the length of the major and the minor axes.

The velocity distribution at various axial locations was obtained by plotting approximately 100 points of the axial components of particle translational velocities (calculated from tracings of the tracer particle paths that were in good focus and appeared to be located in or close to the median plane of the vessel normal to the viewing axis) against the radial distance from the vessel wall. The wall shear stress, $\tau_{\mathrm{w}}=\mu G_{\mathrm{w}}$, was calculated by multiplying the wall shear rate, $G_{\mathrm{w}}$, obtained from the slope of the tangent drawn at the vessel wall on the near-wall velocity distribution by the viscosity of the fluid, $\mu$. We estimate the near wall spatial resolution of the particle velocity measurements was at most one diameter of the largest tracer particle that was $330 \mu \mathrm{m}$.

The wall shear stress obtained above was calculated using only the axial component of velocity. Therefore, strictly speaking, it is not the true but an apparent wall shear stress. We used it as a first approximation of the wall shear stress. Details of the procedures used to obtain velocity distributions and wall shear stresses are given in the Appendix.

The locations of the points of flow separation and stagnation (indicated by $\mathrm{S}$ and $\mathrm{P}$, respectively, in Fig. 3) were determined from the movements of the smallest visible tracer microspheres $(50$ or $80 \mu \mathrm{m}$ diameter particles) that flowed very close to the vessel wall. Throughout the whole flow studies and analyses, special attention was paid to the events occurring near the vessel wall. 


\section{Results}

\subsection{Anatomical structure of the aortic arch}

Figure 2 shows the photographs of several aortic arches prepared in the present study. First, it was found that the aortic arch was not just a curved pipe, but was a tapered vessel with three major side branches coming off the aorta at the apex of the curvature. Secondly, the diameter of the aorta decreased stepwise distal to each side branch. This feature was more remarkable in vessels of young subjects that had fewer and milder atherosclerotic lesions as shown in panel (a). By contrast, in vessels obtained from aged subjects, the proximal descending aorta was dilated as shown in panel (d). Thirdly, the aortic arch was not plane but twisted around the branching site of the left subclavian artery towards the dorsal side of the heart. Thus the descending aorta was located dorsally relative to the ascending aorta. Furthermore, in vessels obtained from aged subjects that had more severe atherosclerotic lesions, the ascending aorta was dilated and elongated as shown in panel (d), making itself convex towards the anterior dorsal wall, as shown in panel (e). By contrast, the proximal descending aorta was convex towards the posterior ventral wall. As a result, the space between the ascending and descending aortas was wider than that of the vessels obtained from young subjects (cf. panels (a) and (d)). Finally, the orifice of the brachiocephalic artery was located not in the common median plane of the aortic arch and its two side branches, that is, the left common carotid artery and the left subclavian artery (will be referred to median plane hereafter) but displaced towards the dorsal side of the heart (cf. panel (c)), even though there was a marked variation in branching patterns of the aortic arch as shown in panels (a), (b) and (d).

It was also noticed that the flow divider of side branches was sharp, and the vessel wall opposite the flow divider was gently rounded. In several vessels, there was no interval between the orifices of the side branches as shown in panel (a). Due to that, the outer wall (the vessel wall located opposite the flow divider) of the left common carotid artery and left subclavian artery served as a part of the flow divider of the brachio-cephalic artery and left common carotid artery, respectively.

\subsection{Preferred sites of atherosclerotic lesions}

Atherosclerotic lesions in the aortic arch were more severe on the ventral wall than on the dorsal wall in thirty-two cases (85\%). As shown in Fig. 2, panels (b), (d) and (e), atherosclerotic plaques were observed frequently on the inner and/or the ventral walls of the aortic arch corresponding to the branching site of the left subclavian artery. The proximal lip of the orifice of each branch was a little more affected by atherosclerosis than the flow divider. However, the aortic wall just distal to the flow divider of the branch was also affected in several vessels as shown in panel (d).

\subsection{Flow patterns in the aortic arch}

In the present study, flow experiments were carried out only under steady flow that simulated aortic flow in mid-systole since the vessel walls of the aortic tree lost their elasticity and became rigid during the process of fixing and rendering them transparent. As a result, it was found that, although the exact nature of the flow was different from one vessel to another due to the variations in the curvature of the arch and branching pattern of the three branches, the general flow patterns, characterized by the formation of a single right-handed helical flow in the arch and a recirculation zone at the inlet of the branches, were qualitatively the same. Given that, and the very laborious and painstaking nature of the work involved in film analysis and preparation of drawings, we analyzed the films that recorded the flow 

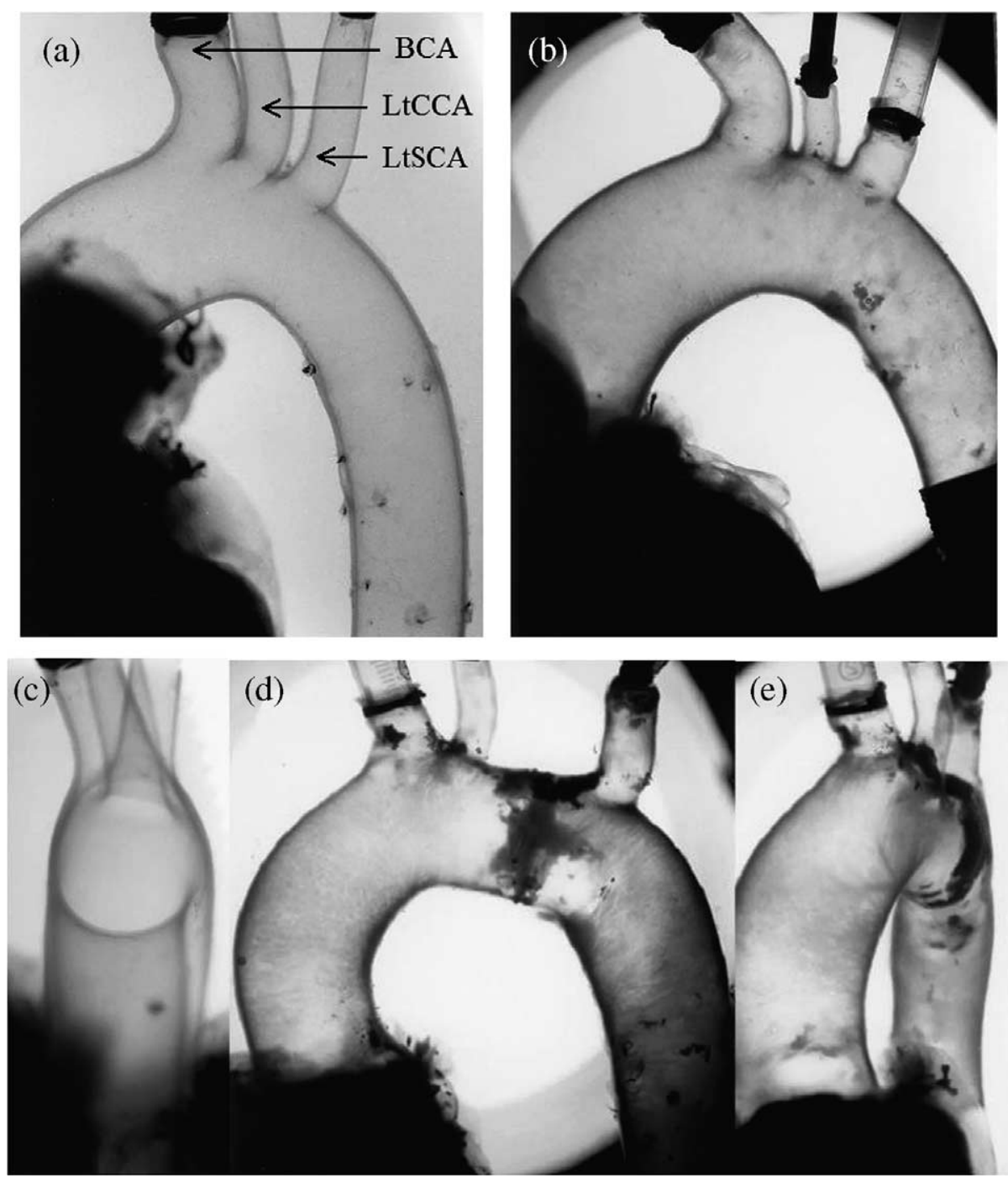

Fig. 2. Photographs of a portion of the aortic arch of several post-mortem human aortic trees, rendered transparent, showing the geometric structure of the aortic arch of a young person in which no atherosclerotic lesions developed in (a) and (c), and of aged persons in which atherosclerotic lesions and calcified plaques developed in (b), (d) and (e).

(a) The arch and its side branches from a 21-year-old female observed normal to common median plane (left anterior oblique view) and (c) observed along the common median plane (right anterior oblique view). Note: aortic diameter decreases stepwise after each branch of the 3 arteries, and orifice and axis of the brachio-cephalic artery (BCA) do not lie in the median plane but are displaced towards the dorsal side of the heart. (b) Left anterior oblique view of arch from a 67-year-old male showing small calcified plaques at the inner wall of the distal arch and proximal descending aorta. (d) Arch from a 63-year-old male in a left anterior oblique view, and (e) frontal view showing severe calcified plaques at (i) the ventral and inner walls of the mid-arch, (ii) right lateral wall of the orifice of the BCA, (iii) proximal lip of the orifice of the left common carotid artery (LtCCA), (iv) the outer wall of the aortic arch between the orifices of the BCA and left subclavian artery (LtSCA), and (v) the proximal lip of the orifice of the LtSCA. Note: in these older men there was no stepwise reduction in aortic diameter and the ascending aorta was deformed in the shape of a convex bulge towards the right anterior wall. The anterio-posterior distance between the ascending and descending aorta is wider than that in the aortic arch shown in panel (a). 
in only one vessel. That vessel was the one we considered the best model prepared in the present study judging from its configuration and the fact that it created minimal flow disturbances at the inlet of the ascending aorta.

Figure 3, panels (a) and (b) show flow patterns represented by particle paths in the aortic arch shown in Fig. 2, panels (a) and (c) that was prepared from a 21-year-old male subject. The former was observed from the ventral wall to the dorsal wall, that is, normal to the median plane of the aortic arch. The latter

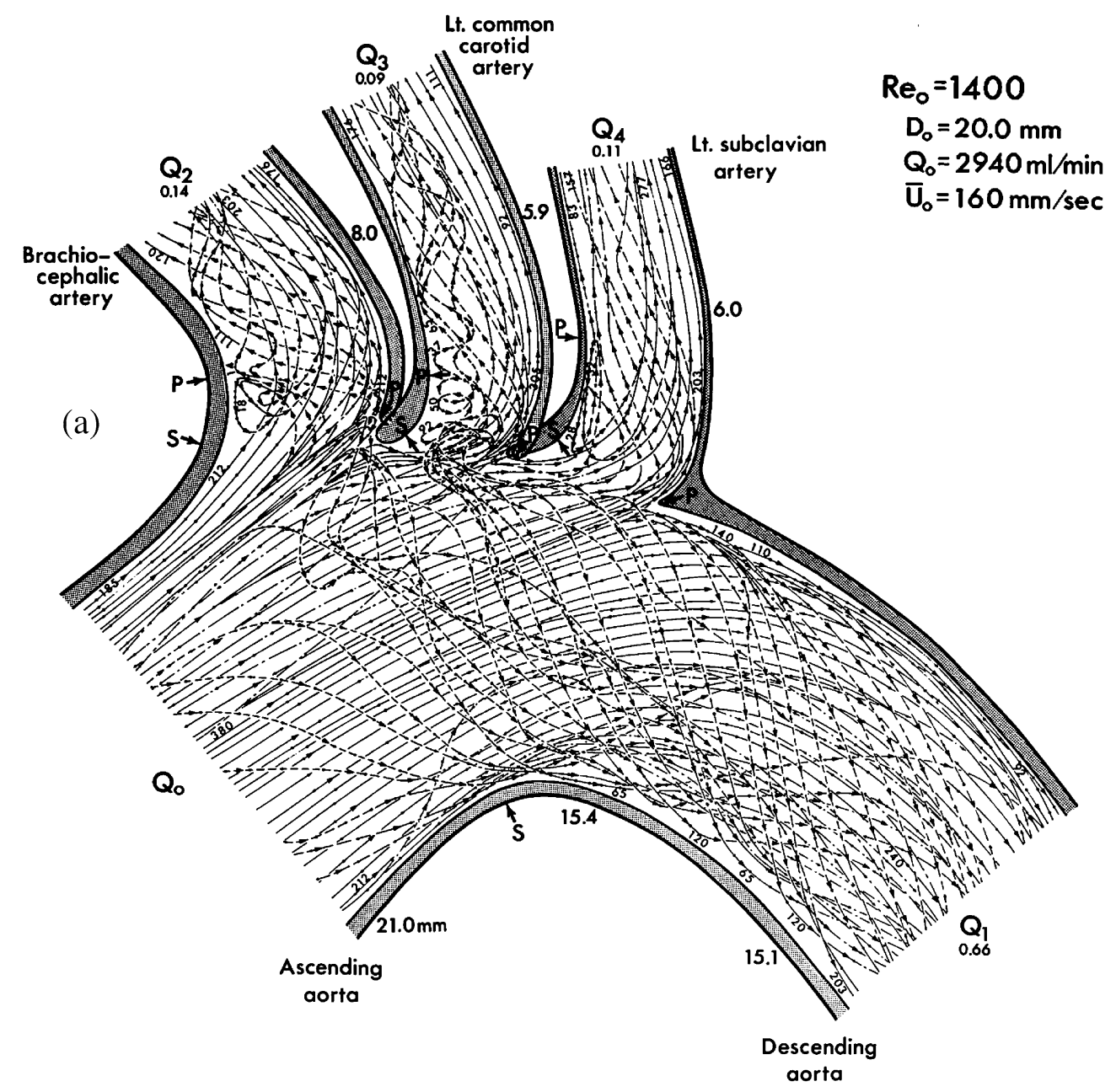

Fig. 3. (a) Detailed flow patterns obtained in steady flow in the transparent human aortic arch from the 21-year-old female shown in Fig. 2(a) and (c), observed normal to the common median plane of the arch and its branches (left anterior oblique view), and (b) along the common median plane (right anterior oblique view). Drawing constructed by tracing paths of tracer microspheres shows a clockwise rotational flow along the ventral wall of the arch and a small recirculation zone at the proximal lip of the orifice (opposite the flow divider) of each branch. Solid lines: paths of particles located in or close to the common median plane; short dashed lines: paths far out of the common median plane; long dashed lines or dash-dotted lines: paths located between the first two types of paths. Arrows at "S" and "P" indicate the points of flow separation and stagnation, respectively. Numbers at the outside of the vessel indicate the inner diameter of the vessel. Numbers along the particle paths indicate particle translational velocities in $\mathrm{mm} / \mathrm{s}$ at positions shown. $Q_{i}(i \geqslant 1)$ : ratio of flow rate in aorta and in the branches to the inflow rate $\left(Q_{0}\right)$. Parameters assessed at inlet of the aortic segment. $\operatorname{Re}_{\mathrm{o}}$ : Reynolds number, $D_{\mathrm{o}}$ : vessel diameter, $Q_{\mathrm{o}}$ : volume flow rate, $\bar{U}_{0}$ : mean fluid velocity. 


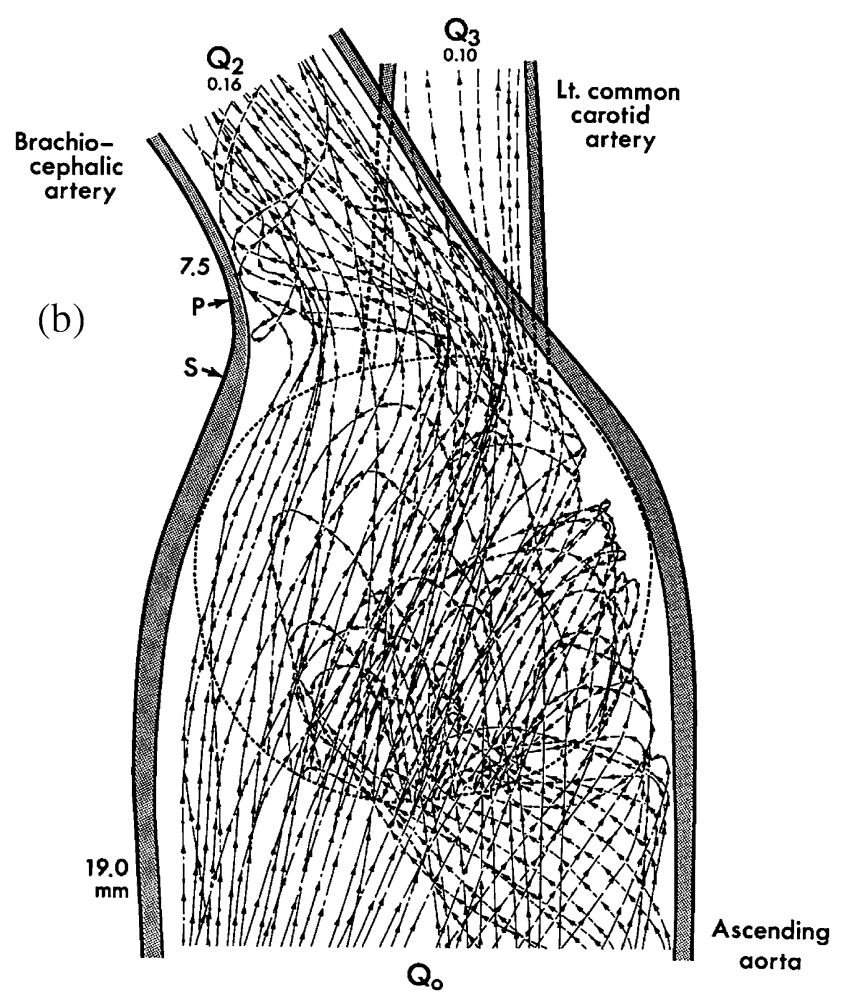

Fig. 3. (Continued.)

was observed from the anterior wall to the posterior wall, that is, along the median plane. In this vessel as well as in all other 3 vessels studied, the orifices and axes of both the left common carotid artery and left subclavian artery were located approximately in the median plane of the aortic arch, while those of the brachio-cephalic artery were located not in the median plane of the aortic arch but displaced towards the dorsal side of the heart as shown in Fig. 2, panel (c) and Fig. 3, panel (b). Due to that as well as the fact that the aortic arch was twisted around the branching site of the left subclavian artery towards the dorsal side of the heart, the flow in the aortic arch was very complex.

The flow in the aortic arch consisted of three major components, namely, a straight flow to the brachiocephalic artery located close to the right dorsal wall of the ascending aorta, a quasi-parallel undisturbed flow located close to the median plane of the aortic arch, and a clockwise rotational flow located dominantly near the left ventral wall of the aortic arch. Thus, looking down the aorta from its origin, the flow in the aortic arch appeared as a single helical flow revolving in the clockwise direction.

\subsection{Detailed structure of the flow in the aortic arch}

The flow ejected from the left ventricle was divided into three groups by the flow behavior of tracer particles.

\subsubsection{The particles on streamlines located on the dorsal side of the median plane}

Particles flowed along the dorsal wall and entered the brachio-cephalic artery and the descending aorta, forming the mainstream in that branch and the descending aorta, respectively. However, due to the 
particular arrangement of the brachio-cephalic artery, that is, both the orifice and the axis of the branch were located not in the median plane but closer to the right dorsal wall of the aortic arch, particles that entered the brachio-cephalic artery were deflected circumferentially on approaching the flow divider of the branch. They traveled along the vessel wall of the branch to the dorsal wall wrapping the mainstream in the branch as shown by the dashed lines, forming a strong single spiral secondary flow. A part of them changed the direction on approaching the dorsal wall and flowed back towards the point of flow separation indicated by the letter S. They then changed the direction again and joined the mainstream in the branch. As a result, a small recirculation zone formed in the region of separated flow as shown in Fig. 3, panels (a) and (b). Flow separation occurred at the dorsal proximal lip of the orifice of the brachio-cephalic artery and at the inner (lower) wall of the mid-arch, as indicated by the letter $\mathrm{S}$ in Fig. 3, panels (a) and (b).

\subsubsection{The particles on streamlines located close to the median plane}

The particles on the anterior part of the streamlines located close to the median plane of the aortic arch directly entered the left common carotid and left subclavian arteries and formed the mainstream in these branches as shown by the solid lines in Fig. 3, panel (a). Flow separation occurred at the proximal lip of the orifice of each branch at the point indicated by the letter S in Fig. 3, panel (a). On entering these branches, the particles were deflected at the flow divider to both sides of the median plane of the aortic arch, due to the fact that the center of the orifice of these branches was located in the median plane of the aortic arch. Then they traveled either along the ventral or dorsal wall wrapping the mainstream in each branch as shown by the dashed lines. A part of them changed the direction on approaching the anterior wall and flowed back towards the point of flow separation indicated by the letter S. They then changed the direction again and joined the mainstream in the branch. As a result, a small recirculation zone formed as shown in Fig. 3, panels (a) and (b). The others in larger orbits formed paired spiral secondary flows along the anterior wall of each branch as shown in Fig. 3, panel (a).

The particles on the posterior part of the streamlines located close to the median plane of the aortic arch passed by the junctions of side branches and traveled almost parallel to the vessel wall and entered the descending aorta. Flow separation occurred at the inner wall (lower wall) at the point indicated by the letter S in Fig. 3, panel (a). In fact, flow separation occurred not at a single point but along a curvilinear line located three-dimensionally along the ventral inner (lower) wall. When they entered the proximal descending aorta, they were deflected at the ventral posterior wall because the proximal descending aorta was twisted towards the dorsal wall. They traveled along the ventral wall as shown by the dashed lines in Fig. 3, panel (a), and were drawn into the region of separated flow formed along the inner wall of the proximal descending aorta.

\subsubsection{The particles on streamlines located on the ventral side of the median plane}

In general, the particles travelled more slowly than those located on the dorsal side. Especially those particles located along the outer and ventral walls of the proximal portion of the aortic arch traveled very slowly along the left ventral wall encircling the mainstream as shown by the dashed lines in Fig. 3, panels (a) and (b) and entered the region of separated flow situated along the inner (lower) wall of the aortic arch distal to the point of flow separation indicated by the letter S in Fig. 3, panel (a). As a result, a single helical flow (spiral secondary flow) revolving in the clockwise direction formed in the aortic arch as shown in Fig. 3, panels (a) and (b). 


\subsection{Distributions of fluid velocity and wall shear stress}

Figure 4, panels (a) and (b) show distributions of fluid axial velocity and wall shear stress (to be referred to velocity distributions hereafter) in the median plane and the diametrical plane normal to the median plane of the aortic arch, respectively, corresponding to the flow shown in Fig. 3. As evident from these figures, the velocity distribution at the proximal aortic arch was blunted and skewed towards the dorsal wall due to the fact that the brachio-cephalic artery (the first and the largest branch) was located on the dorsal side of the median plane of the aortic arch. Throughout the whole aortic arch, it remained almost flat and did not skew towards the outer wall of the arch. The velocity distribution in the branches was skewed towards the posterior (inner) wall as shown in Fig. 4, panel (a). Further more, in the brachio-cephalic artery, it was skewed towards the ventral (left lateral) wall as shown in Fig. 4, panel (b).

The distribution of wall shear stress showed that the ventral wall of the mid-arch, the dorsal-proximal lip of the orifice of the brachio-cephalic artery, and the proximal lip of the orifice of other two branches were exposed to low wall shear stress. Furthermore, although we could not obtain the wall shear stress due to lack of sufficient number of tracer particles in some regions, judging from the velocity of tracer particles shown along the particle paths, the lowest (or very low) wall shear stress was located in the region of the recirculation flow that formed at the proximal lip of the orifice (opposite the flow divider) of each branch. The locations of atherosclerotic lesions formed at the proximal lip of the orifice of three

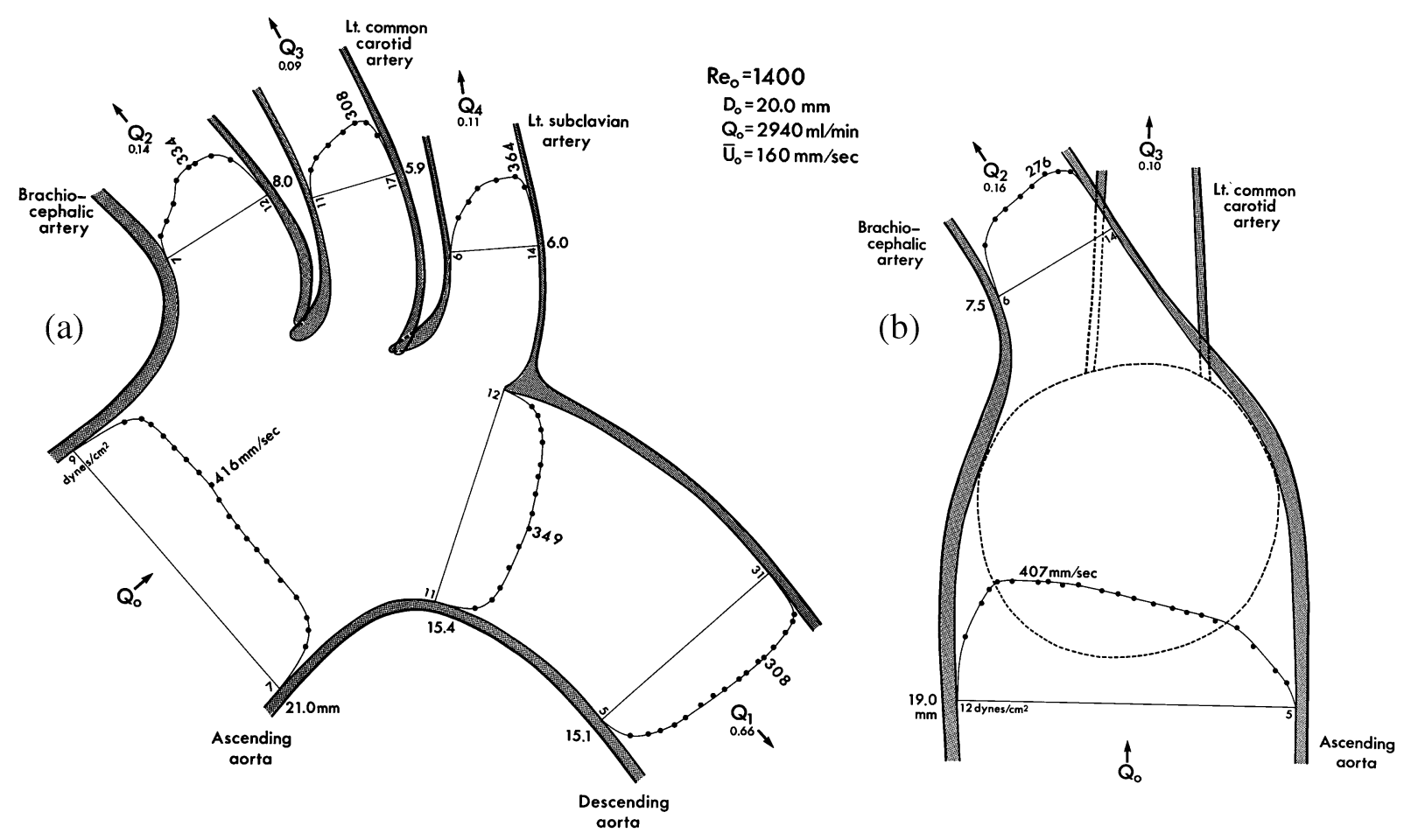

Fig. 4. Distributions of axial fluid velocity and wall shear stress obtained for the flow shown in Fig. 3, illustrating that velocity profiles are almost flat throughout the entire ascending aorta, but skewed towards the dorsal wall in the arch and towards the posterior (inner) wall in the branches. Wall shear stress was low at the ventral wall of the mid-arch and outer wall of each branch. Numbers along velocity profiles give the maximum velocity at the cross-section shown. Numbers at the outside and inside of the vessel show the respective inner diameter of the vessel and wall shear stress measured at each location. 
branches of the aortic arch shown in Fig. 2, panel (d) appear to correspond to these regions of very low wall shear stress.

\section{Discussion}

To carry out the present study, we originally planned to investigate both flow patterns and preferred sites of atherosclerotic lesions using the same vessel. However, it was not possible to do so due to the severity of lesions in most of the vessels prepared from aged subjects. In such a vessel, it was likely that flow patterns were modified by the presence of raised lesions. Therefore, we decided to divide the aortas obtained at autopsy into two groups, namely, one with few lesions for the study of flow patterns and the other with severe lesions for the study of preferred sites of atherosclerotic lesions. By doing so, we could obtain initial flow patterns in the aortic arch before the development of atherosclerotic lesions and study the relationship between the detailed flow patterns obtained and the preferred sites of atherosclerotic lesions observed in vessels with severe lesions. We could also compare the flow patterns obtained with the human aortic arches with those previously obtained with the dog aortic arches [40].

The present study was carried out in relation to the localization of atherosclerotic lesions. Therefore, we focused our study to find out if there were any unusual features in flow patterns directly related to the specific sites of atherosclerotic lesions observed in the aortas obtained at autopsy and used for flow studies. To do so, based on the fact that atherosclerotic lesions develop in relatively large arteries where flow is fast and over a long time, we considered that in the development of atherosclerotic lesions, the average aortic flow rate during systole plays a more important role than the instantaneous rate at peak systole. Therefore, we chose the flow rate characteristic of mid-systole as representative of the aortic flow related to the localization of atherosclerotic lesions.

The flow in the human aortic arch obtained under the steady flow condition was essentially the same as that we observed previously in the dog aortic arch although the number of side branches was different [40]. It was characterized by the formation of a slow single clockwise rotational flow along the ventral wall of the aortic arch and a recirculation flow at the proximal lip of the orifice (opposite the flow divider) of the side branches. It appeared to be determined by three factors, that is, the velocity profile of the outflow from the left ventricle, twisting of the arch, and the arrangement of the three side branches of the aortic arch.

Regarding the velocity profile of the outflow from the left ventricle, Nayler et al. [29] obtained coronal slices of the human heart and the aorta by using a cine-magnetic resonance and showed that the outflow from the left ventricle was skewed towards the dorsal wall of the ascending aorta. Klipstein et al. [25] studied blood flow patterns in ten healthy human aortas by magnetic resonance. They showed that the velocity profile in the ascending aorta during systole was skewed towards the right posterior wall of the vessel. In a similar study carried out using trans-esophageal cardioscopy, Frazin et al. [22] showed the presence of a clockwise rotational flow (helical flow) in the aortic arch and proximal descending aorta in healthy humans. More recently, much clear pictures of the helical flow were presented. Kilner et al. [24] applied time resolved phase contrast magnetic resonance imaging (PC-MRI) to ten healthy volunteers, and investigated the distribution of secondary flows in the aortic arch. They found that right-handed helical flows predominated in the upper aortic arch in late systole in 9 of 10 subjects, although the patterns in the upper descending aorta varied between subjects depending on arch curvature. They concluded that helical and retrograde streams are consistent features of intra-aortic flow in healthy subjects that results, at least in part, from the curvature of the arch and pulsatility of the flow in it. Morbiducci et al. [27,28] 
carried out similar studies by means of 4D PC-MRI. They obtained very clear pictures of right-handed helical blood flow and confirmed that it is an emerging behavior that is common to normal individuals. Bürk et al. [21] and Frydrychowicz et al. [23] studied interdependencies of aortic arch secondary flow patterns, geometry, and age using 4D PC-MRI. They obtained a clear picture of helical flow in systole and concluded that this was a typical pattern in standard crook-shaped aortic arch. However, they observed substantially different flow patterns in the normal thoracic aorta depending on age and diameter of the ascending aorta of the subject.

The results obtained by the use of various diagnostic equipments described above agree well with our findings that the velocity profile at the inlet of the ascending aorta was skewed towards the dorsal wall and a slow single clockwise rotational flow formed along the ventral wall of the aortic arch. However, there is a certain difference between the flow patterns they obtained by the use of 3D and 4D MRI and those we obtained by tracing particle paths. They showed the flow patterns at various times during one cardiac cycle but only superficial pictures of helical and vortex flows that formed within the aortic arch, while we provided detailed information on the structure of the complex flow not only in the aortic arch but also in its side branches. We consider that the results of our present study, showing a right-handed helical flow obtained under steady flow simulating mid-systole, are representative of one systolic segment of the blood flow patterns obtained by Kilner et al. [24], Frydrychowicz et al. [23] and Morbiducci et al. [28,35] using 3D and 4D MRI.

Regarding the topographic distribution of atherosclerotic lesions in the human aortic arch, detailed data are scarce, and the only article we came across is by Yamanaka et al. [20]. Presumably, this is due to the fact that the atherosclerotic lesion that occurs in this region is not so serious compared to that occurs in the abdominal aorta. Yamanaka et al. [20] investigated the prevalence of atherosclerotic lesions in the region of the aortic arch on fifty autopsy specimens by dividing the aortic arch and the adjacent aorta into 9 segments. Their results showed that the degree of atherosclerosis was significantly higher in the arch than the ascending aorta, and it was predominant at the anterior wall of the distal aortic arch, especially the aorta distal to the left subclavian artery, and around the orifice of the brachio-cephalic artery. In our present work, we have studied both the preferred sites of atherosclerotic lesions and the flow in the human aortic arch, and made it clear that atherosclerotic lesions were localized in regions where wall shear stress was very low as previously reported for other parts of human cardiovascular system $[6,10]$. Precise sites of atherosclerotic lesions we observed were the ventral wall of the aortic arch close to the branching site of the left subclavian artery where a slow single clockwise rotational flow formed and the proximal lip of the orifice of each side branch where a recirculation flow formed. It has been shown that dissecting thoracic aneurysm frequently originates around the orifice of the left subclavian artery [41,42]. It is very likely that the dissection starts right from the site of atherosclerotic lesions.

There were several different patterns in branching of the three side branches as shown in Fig. 2 and pointed out in the anatomical studies carried out by Zamir and Sinclair [43]. However, from our own results obtained with the human and dog aortic arches, it did not seem that the flow in the aortic arch was directly affected by the branching pattern. In addition to the branching pattern, it was also noted that the shape of the aortic arch varied very much from one to another. Presumably it was partly due to the decrease in wall elasticity with aging and development of atherosclerotic lesions. Radiological findings by Schlatmann and Becker [44] indicated that the ascending aorta protrudes towards the anterior and dorsal wall, and the aortic diameter of the thoracic aorta markedly increases. We also observed such phenomena in several vessels prepared from aged subjects (cf. Fig. 2, panels (d) and (e)). It is likely that these morphological changes in the aortic arch also promote skewing of velocity distributions towards 
the dorsal wall as shown by the study of velocity distributions carried out by Yearwood and Chandran [45] using a cast of the human aortic arch. Enlargement of aortic diameter will decrease the fluid velocity hence wall shear stress. This may in turn result in the formation of not only a helical flow but also intra-aortic vortices as observed by Bürk et al. [21] and Frydrychowicz et al. [23], and accelerate the development of atherosclerotic lesions.

In some vessels, atherosclerotic lesions were located not only at the proximal lip of the orifice but also at the flow divider of side branches of the aortic arch. This might be due to the anatomical structure that no aortic wall lies between the orifices, and that the stagnation points, where the flow impinges on the flow dividers of the brachio-cephalic and the left common carotid arteries were located close to the separation point as shown by the letter P in Fig. 3, panel (a). In all the cases, preferred sites for the development of atherosclerotic lesions corresponded to the regions of low wall shear stress.

In summary, we have studied the flow patterns and preferred sites of atherosclerotic lesions in the human aortic arch, and found that the flow in the aortic arch consisted of three major components, that is, a straight flow to the brachio-cephalic artery located close to the right dorsal wall of the ascending aorta, a quasi-parallel undisturbed flow located close to the median plane of the aortic arch, and a slow clockwise rotational flow located dominantly near the left ventral wall of the aortic arch. Atherosclerotic lesions were localized in regions of low wall shear stress such as the proximal lip of the orifice of each branch where a slow recirculation flow formed, and the left ventral wall of the aortic arch where a slow spiral secondary flow formed.

\section{Acknowledgements}

This work was carried out while the authors were working in the McGill University Medical Clinic at the Montreal General Hospital, Montreal, Canada, and it was supported by Grant HL-41187 awarded to Dr. Karino from the National Heart, Lung and Blood Institute, National Institute of Health (USA). The authors thank the late Dr. W.P. Duguid of the Department of Pathology at the Montreal General Hospital for his help in obtaining human aortas at autopsy.

\section{Appendix}

\section{A.1. Measurement of the velocity distributions}

One put a tracing paper on the drafting table, and traced the contour of the whole aortic arch from one projected frame of a $16 \mathrm{~mm}$ cine-film. Then, one chose the diametrical plane where a velocity distribution was to be obtained and drew a diametrical line there. The movie was then run for a short time to find a particle that was in good focus and appeared to be located in or very close to the median plane of the vessel normal to the viewing axis. The movie was reversed to the point where the particle first appeared, and then one traced it by marking the particle center frame by frame (the number of frames determined by its velocity) about the same number on both sides of the diametrical line. A line was then drawn that covered all the points traced. If the line did not cross the diametrical line at right angle, then a straight line that passed through the junction of the above two lines and normal to the diametrical line was drawn. Then the end points of the particle path were projected onto this new line to get an axial component of the particle path. After that, the particle path was moved forward so that the rear end point came onto the diametrical line and the projected length of the particle path represented the magnitude of particle axial 
velocity. We followed the same procedure for approximately 100 particles for the large diameter aorta and a smaller number of particles for the smaller diameter branches, and then chose the line (particle path) that was the longest in each division marked on the diametrical line. Using the forward end points of these lines (particle paths) and the two points at the junction of the diametrical line and the inner wall of the vessel where the velocity was assumed to be zero (no slip condition), we drew a best fit curve that covered most of the highest velocity points in each division of the diametrical line. The actual velocity (axial component) of the particle at one point on the diametrical line was obtained by using the length of the particle path together with the number of frames traced (a constant), film speed, and the magnification of the vessel on the tracing paper.

\section{A.2. Measurement of the wall shear stress}

Using the data on axial velocity obtained above, we plotted the axial velocity against the distance from the vessel wall on a large graph paper to prepare an enlarged version of near-wall velocity distribution. If there was a large gap between the velocity points, we traced some more particles near the vessel wall and added the points to fill the gap. Then using a curved ruler we drew a curved line that fitted most of the highest velocity points. Finally, as described in Section 2.3 above, the wall shear stress, $\tau=\mu G_{\mathrm{w}}$, was calculated by multiplying the wall shear rate, $G_{\mathrm{w}}$, obtained from the slope of the tangent drawn at the vessel wall on the best-fit curve of the near-wall velocity distribution by the viscosity of the fluid, $\mu$. We estimate the near-wall spatial resolution of the particle velocity measurements was at most one diameter of the largest particle, that was $330 \mu \mathrm{m}$.

The wall shear stress obtained above was calculated using only axial component of velocity. Therefore, strictly speaking, it is not wall shear stress but an apparent shear stress. We used it as a first approximation of the wall shear stress. The value of the apparent wall shear stress at the outer wall of the arch and the posterior (inner) wall of the three branches, the ventral (left lateral) wall of the brachio-cephalic artery where particle paths are parallel to the vessel wall will be very close to real wall shear stress but not so close at the distal part of the inner wall of the arch and the anterior (outer) wall of the branches where particle paths are not so parallel to the vessel wall.

\section{References}

[1] Armstrong VW, Cremer P, Eberle E, Manke A, Schulze F, Wieland H, et al. The association between serum Lp(a) concentrations and angiographically assessed coronary atherosclerosis: dependence on serum LDL levels. Atheroscler. 1986;62:249-57.

[2] Gerrity RG. The role of the monocyte in atherogenesis: I. Transition of blood-borne monocytes into foam cells in fatty lesions. Am J Pathol. 1981;103:181-90.

[3] Gerrity RG. The role of the monocyte in atherogenesis: II. Migration of foam cells from atherosclerotic lesions. Am J Pathol. 1981;103:191-200.

[4] Ross R, Harker L. Hyperlipidemia and atherosclerosis. Chronic hyperlipidemia initiates and maintains lesions by endothelial cell desquamation and lipid accumulation. Science. 1976;193:1094-100.

[5] Schwenke DC, Carew TE. Initiation of atherosclerotic lesions in cholesterol-fed rabbits: I. Focal increases in arterial LDL concentration precede development of fatty streak lesions. Atheroscler. 1989;9:895-907.

[6] Asakura T, Karino T. Flow patterns and spatial distributions of atherosclerotic lesions in human coronary arteries. Circ Res. 1990;66:1045-66.

[7] Caro CG, Fitz-Gerald JM, Schroter RC. Arterial wall shear and distribution of early atheroma in man. Nature. 1969;223:1159-61.

[8] Cornhill JF, Herderick EE, Stary HC. Topography of human aortic sudanophilic lesions. Monogr Atheroscler. 1990;15:13-9. 
[9] Kjaernes M, Svindland A, Walloe L, Wille SØ. Localization of early atherosclerotic lesions in an arterial bifurcation in humans. Acta Path Microbiol Scand. 1981;A89:35-40.

[10] Ku DN, Giddens DP, Zarins CK, Glagov S. Pulsatile flow and atherosclerosis in the human carotid bifurcation. Positive correlation between plaque location and low and oscillating shear stress. Arteroscler. 1985;5:293-302.

[11] Schwartz CJ, Mitchell RAJ. Observations on localization of arterial plaques. Circ Res. 1962;11:63-73.

[12] Taylor CA, Hughes TJR, Zarins CK. Effect of exercise on hemodynamic conditions in the abdominal aorta. J Vasc Surg. 1999;29:1077-89.

[13] Friedman MH, Bergeron CB, Hutchins GM, Mark FF, Deters OJ. Hemodynamic measurements in human arterial casts, and their correlation with histology and luminal area. J Biomech Eng. 1980;102:247-51.

[14] Liepsch D, Poll A, Stringberger J, Sabbah HN, Stein PD. Flow visualization studies in a mold of the normal human aorta and renal arteries. J Biomech Eng. 1989;111:222-7.

[15] Moore JE Jr, Ku DN, Zarins CK, Glagov S. Pulsatile flow visualization in the abdominal aorta under different physiologic conditions: implications for increased susceptibility to atherosclerosis. J Biomech Eng. 1992;114:1391-8.

[16] Moore JE Jr, Xu C, Glagov S, Zarins CK, Ku DN. Fluid wall shear stress measurements in a model of the human abdominal aorta: oscillatory behavior and relationship to atherosclerosis. Atheroscler. 1994;110:225-40.

[17] Moore JE Jr, Maier SE, Ku DN, Boesinger P. Hemodynamics in the abdominal aorta: a comparison of in vitro and in vivo measurements. J Appl Physiol. 1994;76:1520-7.

[18] Pedersen EM, Sung HW, Burlson AC, Yoganathan AP. Two-dimensional velocity measurements in a pulsatile flow model of the normal abdominal aorta simulating different hemodynamic conditions. J Biomech. 1993;26:1237-47.

[19] Rieu R, Friggland A, Pelissier R. Velocity distribution along an elastic model of human arterial tree. J Biomech. 1985;18:703-15.

[20] Yamanaka K, Miki S, Kusuhara K, Okita Y, Tahata T. The prevalence of atherosclerotic lesions in the aortic arch. Nihon Kyobu Geka Gakkai Zasshi. 1995;43:432-7.

[21] Bürk J, Blanke P, Stanovic Z, Barker A, Russe M, Geiger J, et al. Evaluation of 3D blood flow patterns and wall shear stress in the normal and dilated thoracic aorta using flow-sensitive 4D CMR. J Cardiovasc Magn Reson. 2012;14:84 (11 pp).

[22] Frazin LJ, Lanza G, Vonesh M, Khasho F, Spitzzeri C, McGee S, et al. Functional chiral asymmetry in descending thoracic aorta. Circulation. 1990;82:1985-94.

[23] Frydrychowicz A, Berger A, Rio AM, Russe MF, Bock J, Harloff A, Markl M. Interdependencies of aortic arch secondary flow patterns, geometry, and age analysed by 4-dimensional phase contrast magnetic resonance imaging at 3 Tesla. Eur Radiol. 2012;22:1122-30.

[24] Kilner PJ, Yang GZ, Mohiaddin RH, Firmin DN, Longmore DB. Helical and retrograde secondary flow patterns in the aortic arch studied by three-directional magnetic resonance velocity mapping. Circulation. 1993;88:2235-47.

[25] Klipstein RH, Firmin DN, Underwood SR, Rees RSO, Longmore DB. Blood flow patterns in the human aorta studied by magnetic resonance. Br Heart J. 1987;58:316-23.

[26] Maier SE, Meier D, Boesiger P. Human abdominal aorta: comparative measurements of blood flow with MR imaging and multigated Doppler US. Radiology. 1989;171:487-92.

[27] Morbiducci U, Ponzini R, Rizzo G, Cadioli M, Esposito A, Cobelli FD, et al. In vivo quantification of helical blood flow in human aorta by time-resolved three-dimensional cine phase contrast magnetic resonance imaging. Ann Biomed Eng. 2009;37:516-31.

[28] Morbiducci U, Ponzini R, Rizzo G, Cadioli M, Esposito A, Montevecchi FM, Redaelli A. Mechanistic insight into the physiological relevance of helical blood flow in the human aorta: an in vivo study. Biomech Model Mechanobiol. 2011;10:339-55.

[29] Nayler GL, Firmin DN, Longmore DB. Blood flow imaging by cine magnetic resonance. J Comput Assist Tomogr. 1986;10:715-22.

[30] $\mathrm{Ku} \mathrm{DN}$, Glagov S, Moore JE Jr, Zarins CK. Flow patterns in the abdominal aorta under simulated postprandial and exercise conditions: an experimental study. J Vasc Surg. 1989;9:309-16.

[31] Gallo D, Santis G, Negri F, Tresoldi D, Ponzini R, Massai D, et al. On the use of in vitro measured flow rates as boundary conditions for image-based hemodynamic models of the human aorta: implications for indicators of abnormal flow. Ann Biomed Eng. 2012;40:729-41.

[32] Jin S, Oshinski J, Giddens DP. Effects of wall motion and compliance on flow patterns in the ascending aorta. J Biomech Eng. 2003;125:347-54.

[33] Lantz J, Renner J, Karlsson M. Wall shear stress in a subject specific human aorta-influence of fluid-structure interaction. Int J Appl Mech. 2011;3:759-78.

[34] Liu X, Fan Y, Deng X, Zhan F. Effect of non-Newtonian and pulsatile blood flow on mass transport in the human aorta. J Biomech. 2011;44:1123-31.

[35] Morbiducci U, Ponzini R, Gallo D, Bignardi C, Rizzo G. Inflow boundary conditions for image-based computational hemodynamics: impact of idealized versus measured velocity profiles in the human aorta. J Biomech. 2013;46:102-9. 
[36] Shahcheraghi N, Dwyer HA, Cheer AY, Barakat AI, Rutaganira T. Unsteady and three-dimensional simulation of blood flow in the human aortic arch. J Biomech Eng. 2002;124:378-86.

[37] Tang BT, Cheng CP, Draney MT, Wilson NM, Tsao PS, Herfkens RJ, Taylor CA. Abdominal aortic hemodynamics in young healthy adults at rest and during lower limb exercise: quantification using image-based computer modeling. Am J Physiol Heart Circ Physiol. 2006;291:H668-76.

[38] Taylor CA, Hughes TJR, Zarins CK. Finite element modeling of three-dimensional pulsatile flow in the abdominal aorta: relevance to atherosclerosis. Ann Biomed Eng. 1998;26:975-87.

[39] Karino T, Motomiya M. Flow visualization in isolated transparent natural blood vessels. Biorheology. 1983;20:119-27.

[40] Endo S, Sohara Y, Karino T. Flow patterns in the dog aortic arch under a steady flow condition simulating mid-systole. Heart Vessels. 1996;11:180-91.

[41] DeSanctis RW, Doroghazi PM, Austen G, Buckley MJ. Medical progress - aortic dissection. New Eng J Med. 1987;317:1060-7.

[42] Slater EE, DeSanctis RW. The clinical recognition of dissecting aortic aneurysm. Am J Med. 1976;60:625-33.

[43] Zamir M, Sinclair P. Continuum analysis of common branching patterns in the human arch of the aorta. Anat Embryol. 1990;181:31-6.

[44] Schlatmann TJ, Becker AE. Histologic changes in the normal aging aorta: implication for dissecting aortic aneurysm. Am J Cardiol. 1977;39:13-20.

[45] Yearwood TL, Chandran KB. Experimental investigation of steady flow through a model of the human aortic arch. J Biomech. 1980;13:1075-88. 